\title{
THE ALIGNMENT OF THE LHCB
}

VERTEX DETECTOR:

\section{PERFORMANCE IN RUN 2 AND STUDIES}

\section{FOR THE UPGRADE}

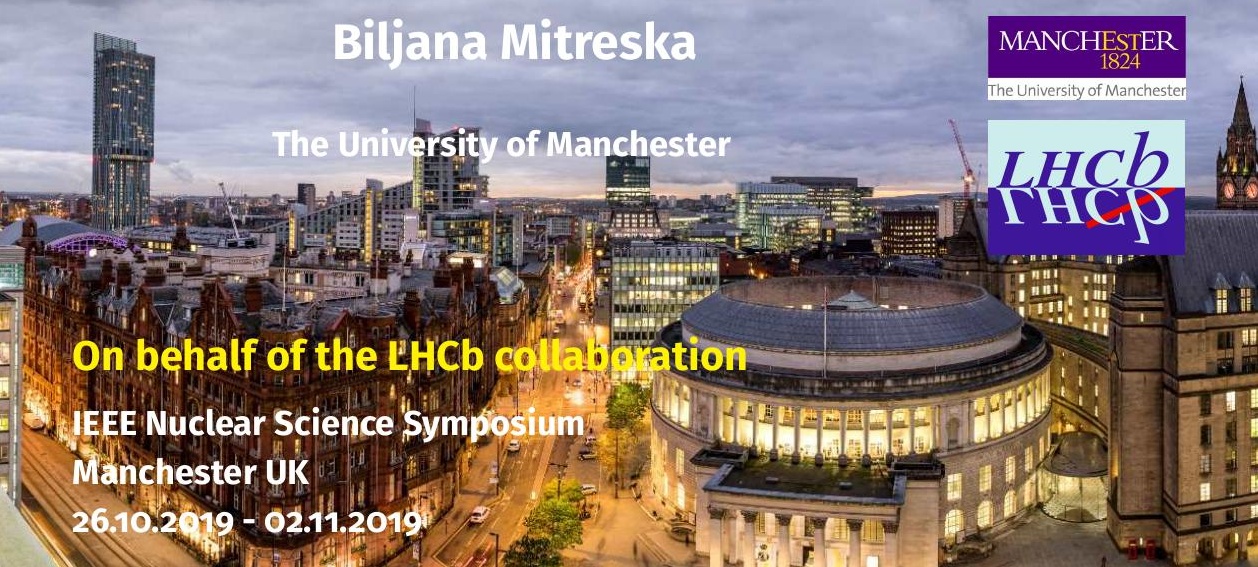




\section{OUTLINE}

1 The LHCb experiment

- The LHCb Vertex Locator (VELO)

2 The alignment

- Real time alignment

3 Performance during Run 2

4 Getting ready for Run 3

- Run 3 alignment 
THE LHCB EXPERIMENT 


\section{THE LHCB EXPERIMENT}

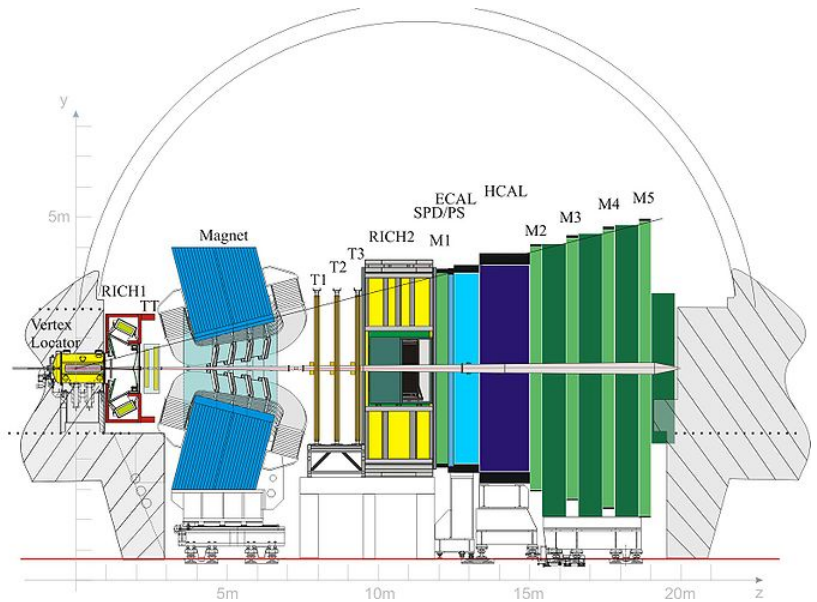

- Forward arm spectrometer

- Measuring decay properties of beauty and charm hadrons

- Tracking system (VELO, TT, IT, OT)
- Calorimeter (ECAL, HCAL)

- Particle identification (RICH, Muon system)

- Operated until the end of Run 2 (2015-2018) 


\section{THE LHCB VERTEX LOCATOR (VELO)}

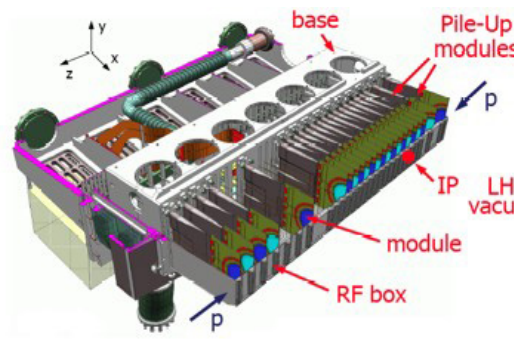

- Silicon strip detector around the LHCb luminous region

- Provides primary and secondary vertex reconstruction

- Precise lifetime measurements

- 42 modules arranged perpendicularly to the beam.

- The sensors have radial geometry
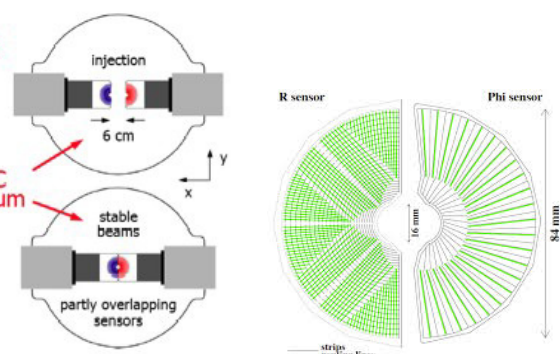

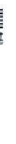

- Two movable halves

- Operating in a secondary vacuum with bi-phase $\mathrm{CO}_{2}$ cooling at temperature $-30^{\circ}$

- This detector operated by the end of 2018

- Now $\rightarrow$ Upgrade I (2019-2021)

- Completely new detector! 
THE ALIGNMENT 


\section{THE ALIGNMENT}
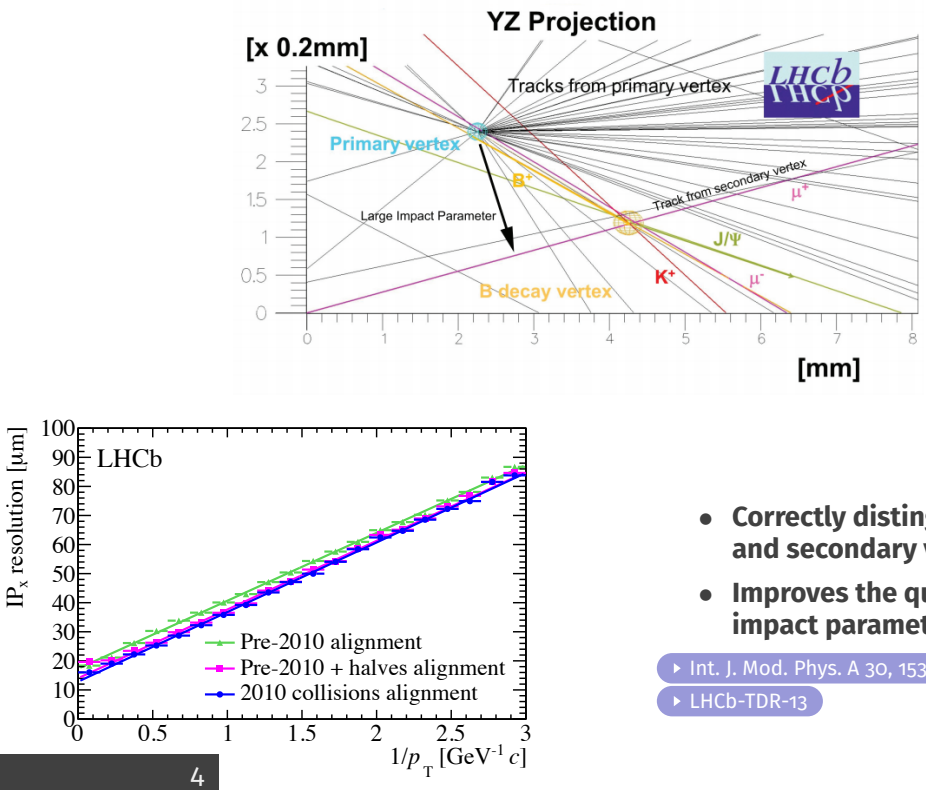

- Correctly distinguishing primary and secondary vertices

- Improves the quality of the impact parameter

Int. J. Mod. Phys. A 30, 1530022 (2015)

- LHCb-TDR-13 


\section{THE ALIGNMENT PROCEDURE}

- Method based on Kalman filter track fit

- Takes a sample of reconstructed tracks

- Minimum $\chi^{2}$ algorithm $\rightarrow$ determine the position of the detector elements

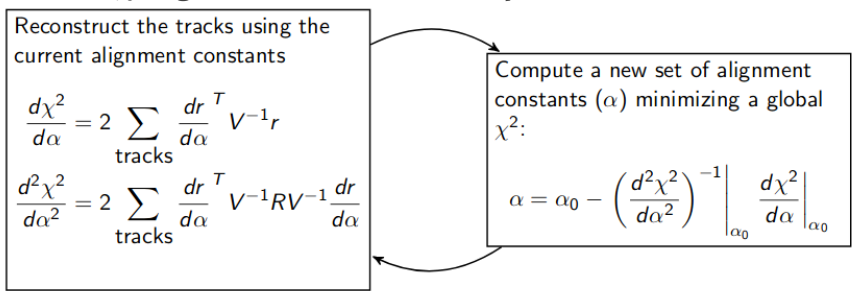

Iterate until the $\chi^{2}$-difference is below a threshold

$r$ : tracks residuals, $V$ : covariance matrix, $R$ : residuals' covariance matrix

- Alignment for 6 degrees of freedom : translations $\left(T_{x}, T_{y}, T_{z}\right)$ and rotations $\left(\mathrm{R}_{\mathrm{x}}, \mathrm{R}_{\mathrm{y}}, \mathrm{R}_{\mathrm{z}}\right)$

- Vertex and mass constraints can be used

- VELO halves and modules can be aligned 


\section{DATA TAKING STRATEGY}

- Run 2 real time analysis model developed by LHCb $\rightarrow$ new trigger model

- Events buffered on disk while performing real-time alignment and calibration

- Physics analysis performed directly from the trigger output (Turbo)

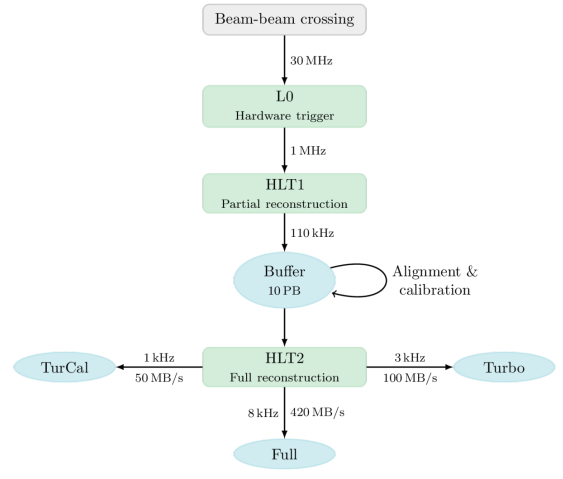

- no offline processing in Run 2

Run 1

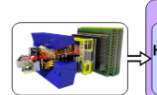

'Online': Near-detector resources

Time from collision: Hardware trigger
$40 \mathrm{MHz} \rightarrow 1 \mathrm{MHz}$
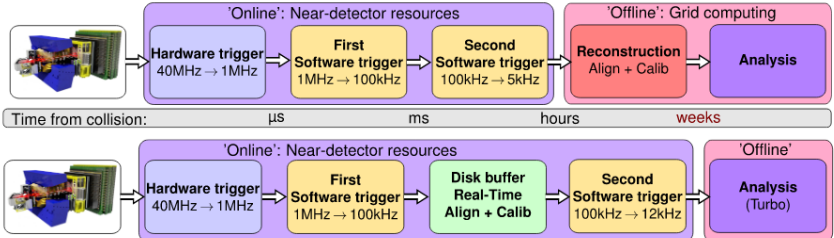
hours weeks

Run 2

Time from collision:

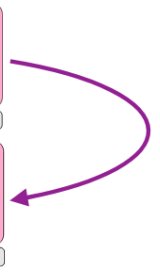




\section{REAL TIME ALIGNMENT}

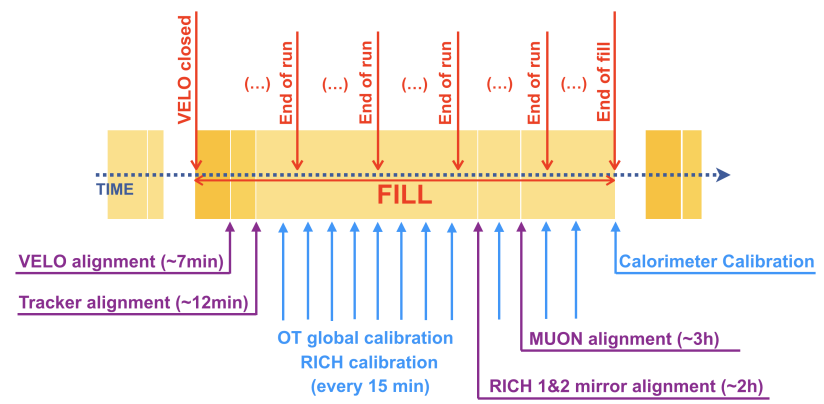

(( $7 \mathrm{~min}),(\sim 12 \mathrm{~min}),(\sim 3 \mathrm{~h}),(\sim 2 \mathrm{~h}))$ - time needed for both data accumulation and running the task

\section{S. Borghi $\rightarrow$ JINST 14 (2019) P04013}

- Run automatically at the start of each fill

- Selecting a dedicated data sample

- Compute the new alignment constants in few minutes

- Automatic update if the variations are significant

VELO alignment tresholds

\begin{tabular}{|cc|}
\hline dof & Threshold \\
\hline \hline $\mathbf{T}_{\mathbf{x}}, \mathbf{T}_{\mathbf{y}}(\mu \mathbf{m})$ & $\mathbf{1 . 5}$ \\
\hline $\mathbf{T}_{\mathbf{z}}(\boldsymbol{\mu} \mathbf{m})$ & $\mathbf{5}$ \\
\hline $\mathbf{R}_{\mathbf{x}}, \mathbf{R}_{\mathbf{y}}(\mu \mathrm{rad})$ & 4 \\
\hline $\mathbf{R}_{\mathbf{z}}(\boldsymbol{\mu} \mathbf{r a d})$ & 30 \\
\hline
\end{tabular}

- The new constants are used in full reconstruction 


\section{PERFORMANCE DURING RUN 2}

\section{Alignment Stability}
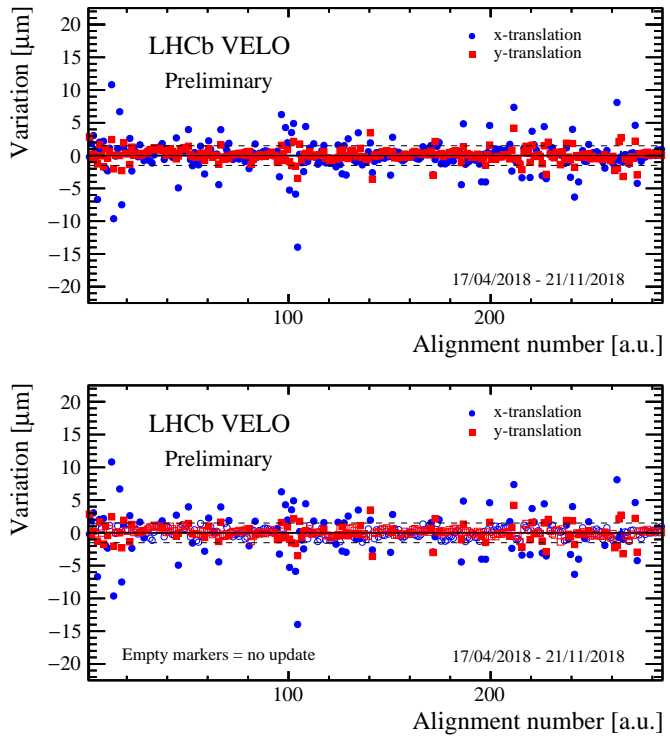

- Each point shows the difference between the initial alignment constants and the new ones

- Stable VELO alignment with update of constants every few fills 


\section{PERFORMANCE DURING RUN 2}

\section{Track reconstruction efficiency}

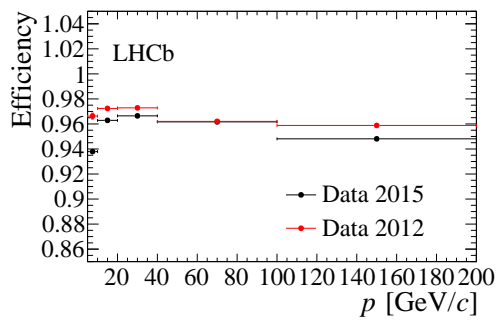

Impact Parameter resolution

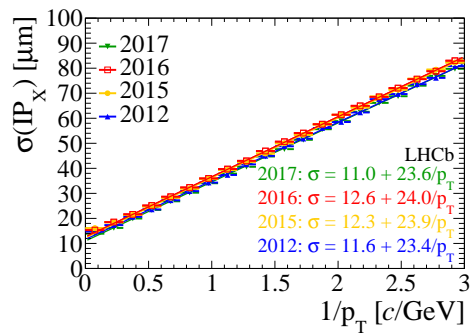

Decay time resolution

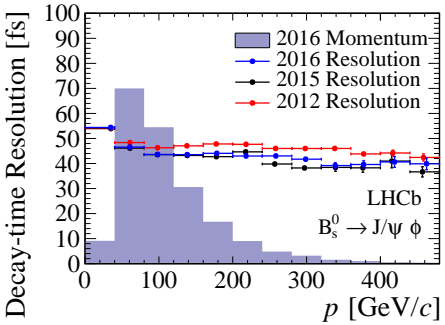

$\mathrm{PV} z$ resolution vs number of tracks

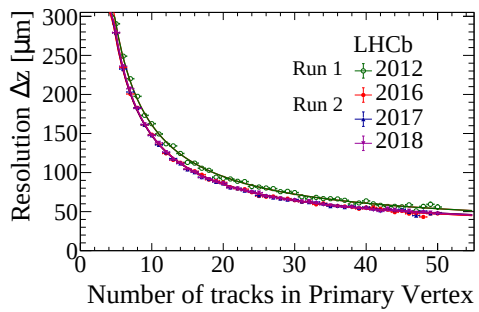




\section{PERFORMANCE DURING RUN 2}

Temperature dependency
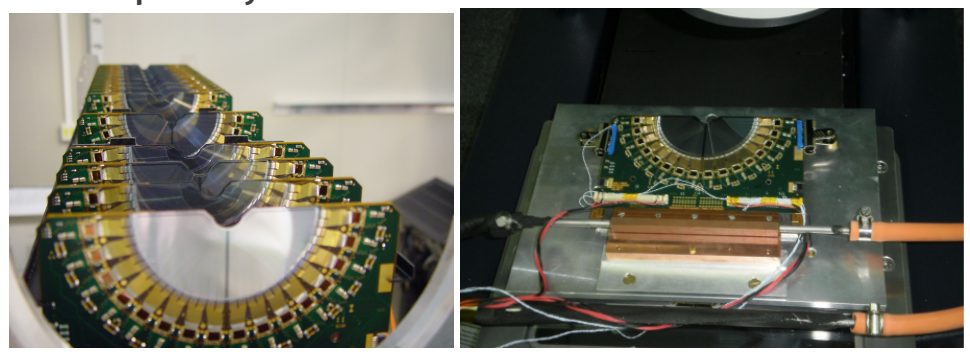

- The VELO operates at $-30^{\circ}$

- The modules' shrinkage at different temperatures that has been measured in lab conditions

- At the end of Run 2 a test with a temperature scan from $-30^{\circ}$ to $-20^{\circ}$ with 4 stages $\left(-30^{\circ},-26^{\circ},-24^{\circ},-20^{\circ}\right)$ was made

- Data samples, taken at each temperature of the VELO, are used to study the detector movements as function of the temperature

- Alignment was evaluated using data at the $\mathbf{4}$ heating stages

- Different alignment scenarios are used 


\section{PERFORMANCE DURING RUN 2}

\section{Temperature dependency}

Halves alignment

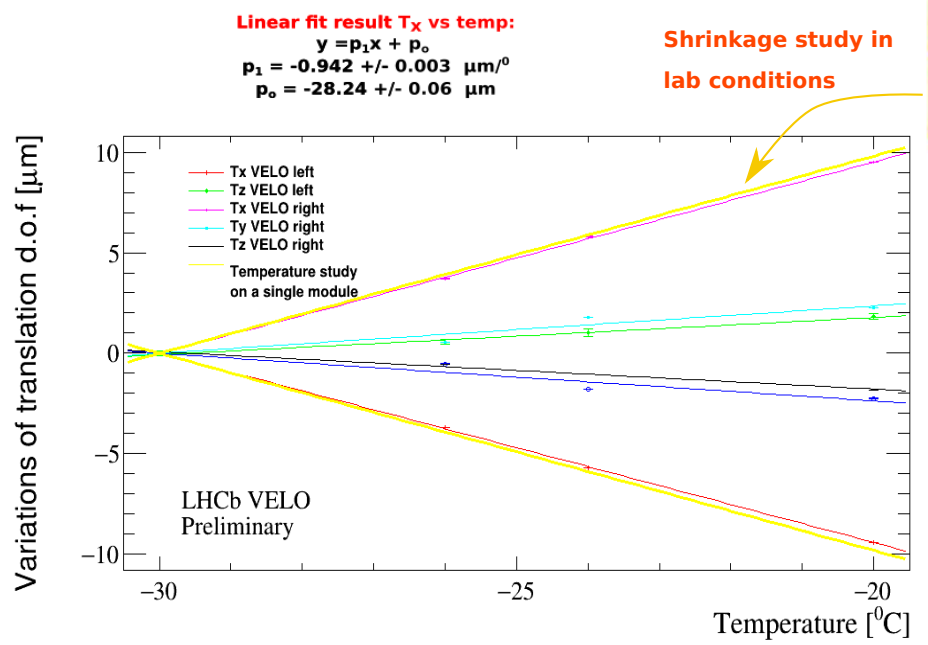




\section{PERFORMANCE DURING RUN 2}

\section{Temperature dependency}

- Variations of the alignment constants wrt $-30^{\circ}$ are calculated

- Linear dependence in temperature $\left(\mathrm{T}_{\mathrm{x}}\right)$

- Variations for $T_{x}$ are largest with $1 \mu \mathrm{m}$ per degree

- Comparison with measurements on a single module

- Studies consistent!

Modules alignment
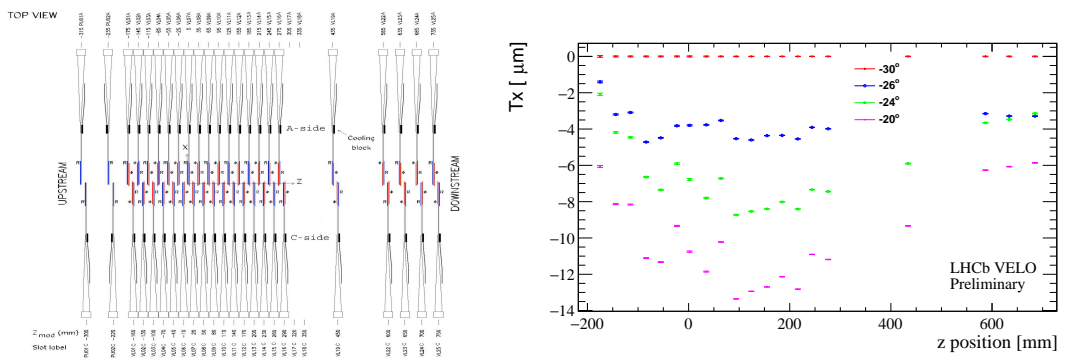

- Variations vs z position of modules are analyzed

- Largest variation temperature $-\mathbf{2 0}{ }^{\circ}$ 


\section{GETTING READY FOR RUN 3}




\section{GETTING READY FOR RUN 3 (2021-2023)}

\section{The Run 3 VELO}

- VELO pixel detector

- $55 \times 55 \mu \mathrm{m}^{2}$ pixels

- 52 modules

- 4 sensors at each module

- 3 Velopix ASICs for each sensor

- $40 \mathrm{MHz}$ readout

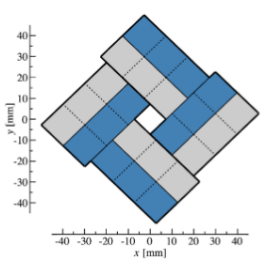

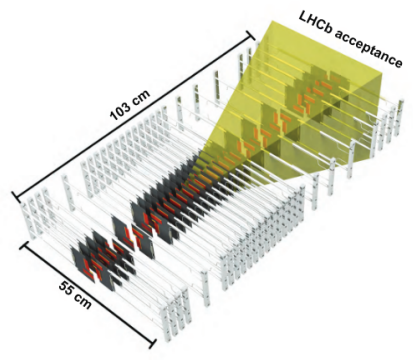

LHCb-TDR-13 more details P.Svihra poster at IEEE Improved efficiencies and IP resolution
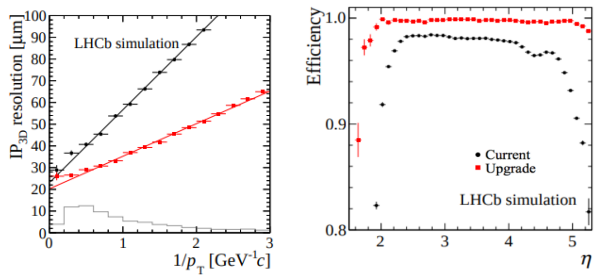


\section{RUN 3 ALIGNMENT PROCEDURE}

- Real time procedure

- Kalman filter

- Using the VeloPix geometry

- Alignment for halves, modules and sensors

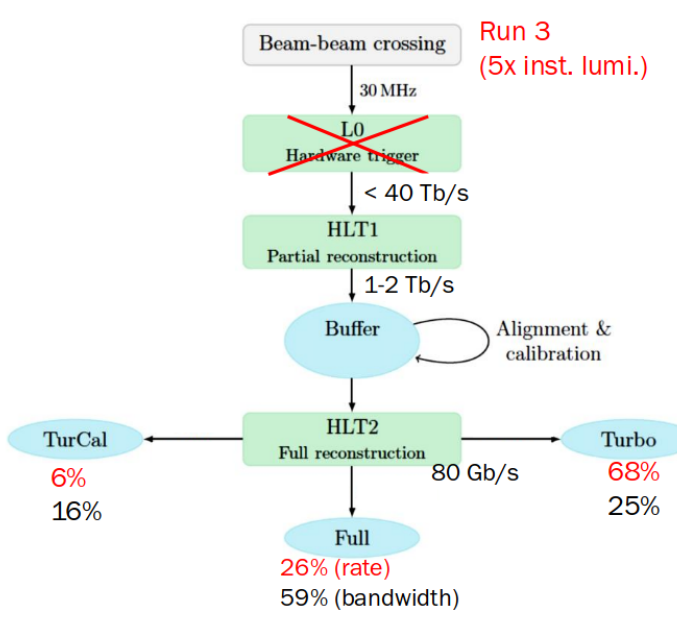




\section{RUN 3 ALIGNMENT STUDIES}

- Starting from misaligned detector (applying large misalignment!)

First look at the alignment for the VeloPix
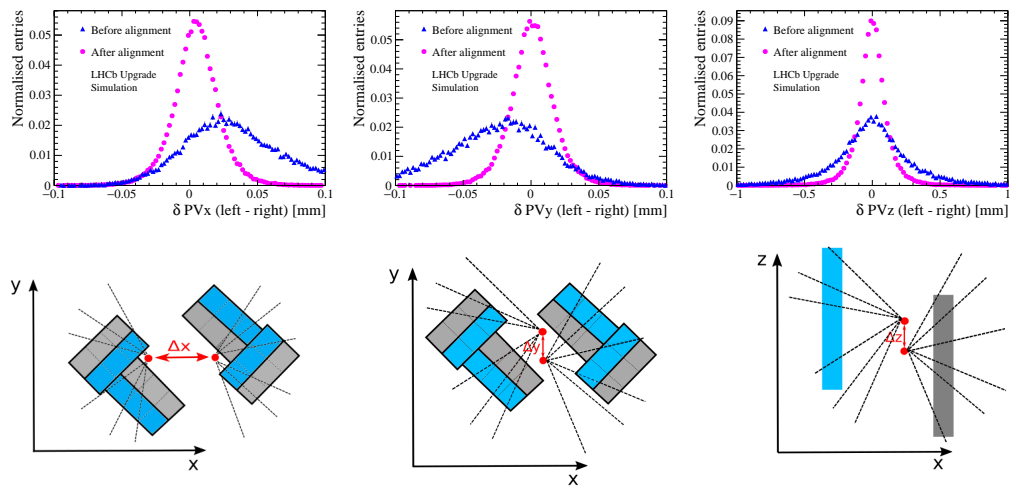

- Distance between $x / y / z$ positions of the reconstructed primary vertices reconstructed with the tracks in each VELO half

- The procedure finds correction of the misalignment applied 
- New strategy for the software trigger has been developed that allowed the alignment and calibration to be performed in real-time

- Alignment of the detector in few minutes

- Same online-offline reconstruction and performance

- Physics analysis possible from the trigger output

- Within Run 2 the alignment of the LHCb VELO proved to be stable

- The variation of alignment constants at different temperatures has been studied

- A first look in the Run 3 alignment procedure is made Thank you! 


\section{RECONSTRUCTION AND PARTICLE TYPES}
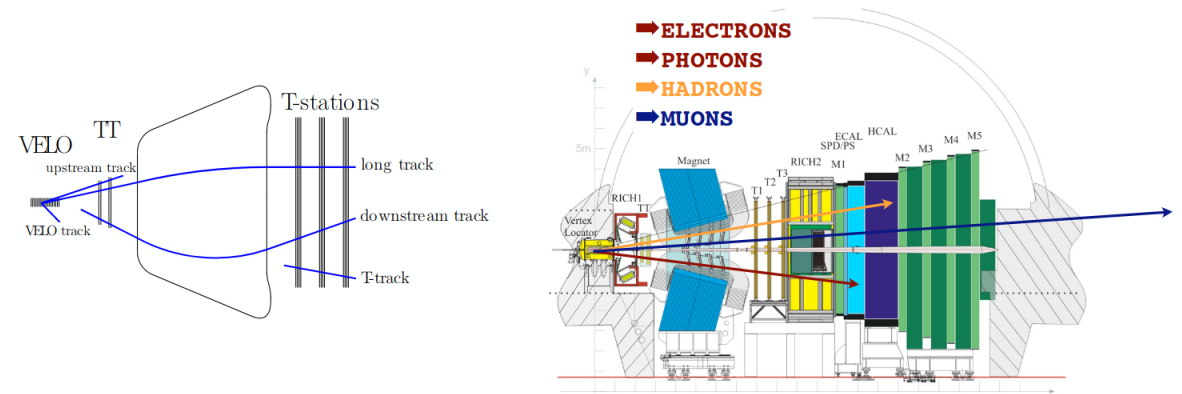


\section{TRIgGER StRATEgy IN RUN 1 (2009-2013)}

LHCb Run I trigger diagram

$15 \mathrm{MHz}$ bunch crossing rate

W
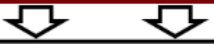

Lo Hardware Trigger : $1 \mathrm{MHz}$ readout, high $\mathrm{E}_{\mathrm{T}} / \mathrm{P}_{\mathrm{T}}$ signatures

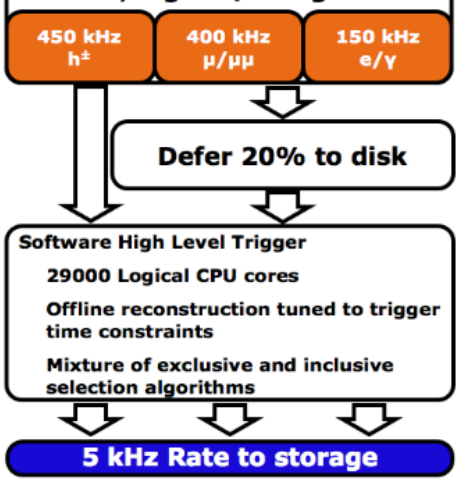




\section{ALIGNMENT STABILITY}
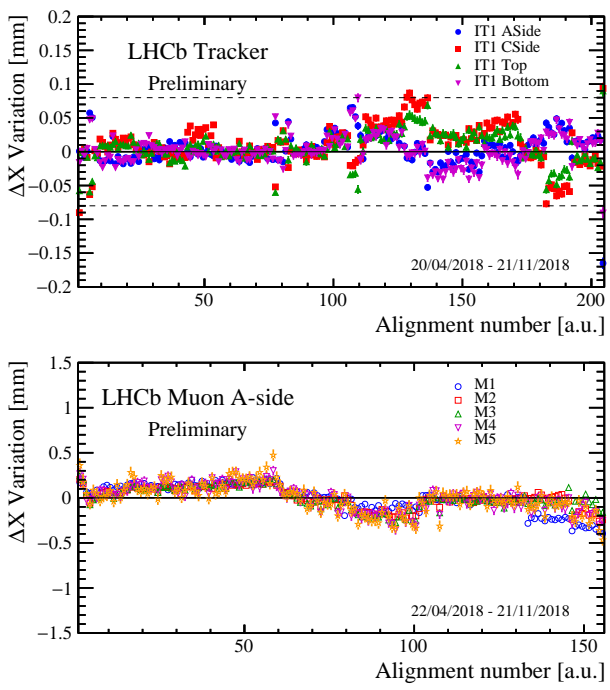

> LHCb-FIGURE-2019-015 


\section{LHCB UPGRADED DETECTOR}

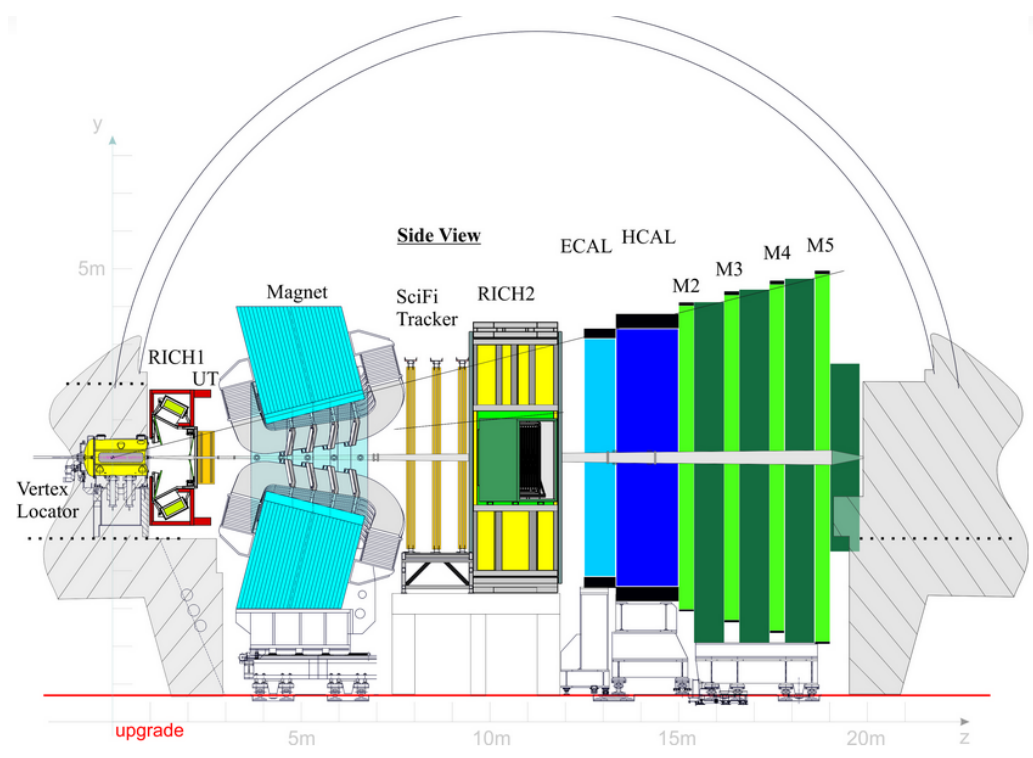




\section{VELOPIX DETECTOR}

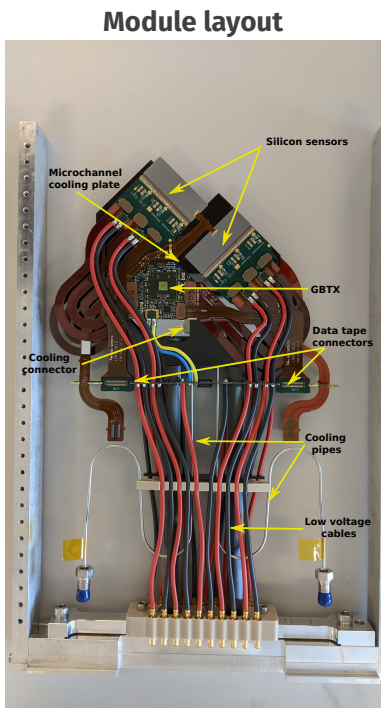

Comparison of VELO z-layouts

— Current

Upgrade

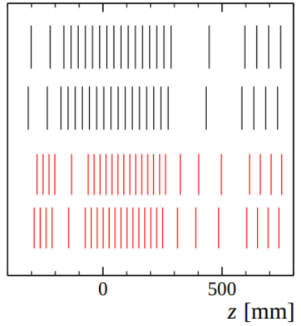

Module at testbeam at CERN

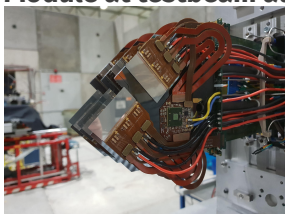

